\title{
Respostas cardiopulmonares durante o esforço em crianças e adolescentes nascidas prematuras
}

\author{
Cardiopulmonary outcomes during stress in children and adolescents born prematurely
}

Josy Davidson ${ }^{1}$, Priscila Cristina João², Regiane de Oliveira Rodrigues ${ }^{3}$, Ana Silvia Scavacini ${ }^{4}$

\section{RESUMO}

Objetivo: Descrever as respostas cardiopulmonares durante o esforço em crianças e adolescentes nascidos prematuros.

Fontes de dados: Busca nas bases científicas em saúde SciELO, Lilacs e PubMed, utilizando-se os descritores: "pressão arterial”, "capacidade física”, "cardiovascular", "prematuro", "criança”, adolescente”, "função pulmonar", nos idiomas inglês e português. Foram selecionados artigos publicados nos últimos 20 anos.

Síntese dos dados: Crianças e adolescentes com histórico de prematuridade apresentam sinais de obstrução das vias aéreas, menores valores de função pulmonar e de capacidade ao exercício, maior incidência de hipertensão arterial e/ou valores mais elevados da pressão arterial sistólica do que os nascidos a termo. Além disso, há indícios de que as alterações na resistência vascular sistêmica desde os primeiros dias de vida comprometam o desenvolvimento cardiovascular até a idade adulta, predispondo a maiores riscos cardiovasculares.

Conclusões: O esforço físico nos indivíduos nascidos prematuros acarreta respostas cardiopulmonares diferentes dos nascidos a termo, atribuídas a particularidades no desenvolvimento desses sistemas inerentes ao prematuro.

Palavras-chave: prematuro; sistema cardiovascular; técnicas de diagnóstico do sistema respiratório.

\section{ABSTRACT}

Objective: To describe cardiopulmonary outcomes during physical stress in prematurely born children and adolescents.

Data sources: Studies were obtained from PubMed, SciELO and Lilacs in Portuguese and in English from the last 20 years. The following key-words were searched: "arterial pressure", "physical capacity", "cardiovascular", "premature", "children", "adolescent" and "pulmonary function".

Data synthesis: Prematurely born children and adolescents have airway obstruction, worse lung function and exercise capacity, more frequency of hypertension and/or higher systolic arterial pressure than those born at term. Furthermore, studies suggest that these infants have cardiovascular resistance alterations since their first days of life that could endanger the development of this system until adulthood, increasing cardiovascular risks.

Conclusions: Physical stress in prematurely born individuals may lead to different cardiopulmonary outcomes compared to those born full-term. These alterations could be attributed to peculiarities of the cardiopulmonary system development.

Key-words: infant, premature; cardiovascular system; diagnostic techniques, respiratory system.
Instituição: Disciplina de Pediatria Neonatal do Departamento de Pediatria da Universidade Federal de São Paulo (Unifesp), São Paulo, SP, Brasil

'Doutora em Ciências pela Unifesp; Tutora da Residência Multiprofissional da Unifesp, São Paulo, SP, Brasil

${ }^{2}$ Especialista em Fisioterapia Respiratória Pediátrica e Neonatal pela Unifesp; Fisioterapeuta da Unidade de Terapia Intensiva Neonatal do Hospital São Paulo da Unifesp, São Paulo, SP, Brasil

${ }^{3}$ Especialista em Fisioterapia Respiratória Pediátrica e Neonatal pela Unifesp; Fisioterapeuta da Unidade de Terapia Intensiva Neonatal do Hospital de Carapicuiba, São Paulo, SP, Brasil

${ }^{4}$ Mestre em Ciências pela Unifesp; Tutora da Residência Multiprofissional da Unifesp, São Paulo, SP, Brasil
Endereço para correspondência:

Josy Davidson

Rua Dr. Diogo de Faria 764

CEP 04037-002 - São Paulo/SP

E-mail: josydavidson@yahoo.com.br

Conflito de interesse: nada a declarar

Recebido em: 12/2/2010

Aprovado em: 23/8/2010 


\section{Introdução}

No decorrer das últimas décadas, o desenvolvimento nos cuidados intensivos neonatais vem proporcionando aumento significativo da sobrevida de recém-nascidos pré-termo (RNPT). As condições pulmonares dos RNPT entre as décadas de 1970 e início da década de 1990, hoje adultos jovens e adolescentes que apresentavam lesões pulmonares típicas da displasia broncopulmonar (DBP), foram se alterando com o avanço científico-tecnológico neonatal dando lugar à "nova DBP”, caracterizada por lesões pulmonares mais amenas e sem relação com o uso de altas pressões inspiratórias e concentrações de oxigênio ${ }^{(1,2)}$.

Dentre os principais fatores pré-natais que determinam o desenvolvimento pulmonar fetal estão o crescimento fetal e a duração da gestação. No entanto, é difícil diferenciar os danos pulmonares decorrentes da prematuridade, isoladamente, daqueles secundários às intervenções sofridas no período neonatal ${ }^{(3)}$, pois ambos estão intimamente relacionados. Fatores como hipoxemia, insuficiência placentária e tabagismo também parecem afetar diretamente o padrão normal de crescimento e de desenvolvimento pulmonar.

O sistema cardiovascular de ex-prematuros também apresenta diferenças em relação a indivíduos nascidos a termo, porém nem todas são conhecidas, principalmente em relação à tolerância a situações de esforço, como no exercício. Portanto, o objetivo desta revisão foi analisar as respostas cardiovasculares e pulmonares de crianças e adolescentes nascidas prematuras durante o esforço.

Para concretizar esse objetivo, utilizaram-se os descritores: "pressão arterial”, "capacidade física”, "cardiovascular”, "prematuro", "criança”, "adolescente”, "função pulmonar”, nos idiomas inglês e português, nas bases científicas SciELO, Lilacs e PubMed, sendo selecionados os artigos publicados nos últimos 20 anos, preferencialmente dos últimos 10 anos, incluindo-se também trabalhos clássicos referentes ao tema, publicados anteriormente.

\section{Respostas ventilatórias ao exercício}

Durante a atividade física em indivíduos normais, independentemente da idade, o crescimento das necessidades metabólicas eleva a ventilação pulmonar à custa do aumento do volume minuto (VE), ou seja, da frequência respiratória (FR) e/ou do volume corrente (VC) e, dessa forma, o coeficiente respiratório é mantido. Em adultos, o incremento de VE decorre do maior $\mathrm{VC}^{(4)}$, diferentemente das crianças que, devido à menor eficiência ventilatória, apresentam importante elevação da FR e respiração mais superficial frente ao maior consumo de oxigênio $\left(\mathrm{VO}_{2}\right)$. Com o avançar da idade, a FR mostra um declínio progressivo e o VC aumenta linearmente, estabilizando-se na maturidade ${ }^{(5)}$. Certamente, os valores proporcionalmente mais elevados do VE na população pediátrica estão relacionados aos níveis de maturação somática, tendo uma relação direta com o crescimento do sistema pulmonar ${ }^{(4,5)}$.

Crianças em idade escolar, com histórico de prematuridade, em condições de estresse aeróbio, apresentam redução do $\mathrm{VE}$, da saturação arterial de oxigênio e do $\mathrm{VO}_{2}$, além de aumento da pressão parcial de gás carbônico ${ }^{(6,7)}$, sendo que, em crianças com diagnóstico de displasia broncopulmonar (DBP) e nascidos entre 24 e 28 semanas de gestaçãa $^{(8)}$, essas ocorrências são ainda mais exuberantes. $\mathrm{Na}$ tentativa de compensar as necessidades aeróbias, há aumento exagerado da FR, associada à manutenção do VC, levando a um VE menor do que o esperado, caracterizando a menor reserva ventilatória ${ }^{(8-10)}$. Um estudo realizado em crianças com histórico de DBP demonstrou que, durante o exercício, elas utilizam até $93 \%$ da sua reserva ventilatória, enquanto nas do grupo controle, nascidas a termo, isso correspondeu a $59 \%$ (7).

A menor reserva ventilatória evidenciada nessa população deve-se à redução do número de alvéolos, desarranjo de tecido elástico e fibroso e hiperdistensão alveolar, ou seja, um padrão discordante entre o crescimento e o desenvolvimento pulmonar e das vias aéreas, levando a alterações clínicas e funcionais ${ }^{(10,11)}$.

A significativa diminuição da função pulmonar nos três primeiros anos de vida nas crianças com DBP observadas pelo aumento da capacidade residual funcional (CRF) e do volume residual (VR), quando comparadas com RNPT sem $\mathrm{DBP}^{(11,12)}$, predispõe à hiperinsuflação pulmonar. Com o avançar da idade, o crescimento do parênquima pulmonar e a multiplicação alveolar propiciam melhora progressiva dos volumes e capacidades pulmonares ${ }^{(13,14)}$, permanecendo, entretanto, abaixo dos valores das crianças nascidas a termo ${ }^{(13,14)}$ pois, mesmo com o crescimento pulmonar, as alterações persistem, demonstrando menor desenvolvimento alveolar e capilar pulmonar ${ }^{(8,12,14-16)}$.

Até a primeira década, as crianças com DBP apresentam redução dos fluxos expiratórios ${ }^{(17-20)}$, com diminuição do volume expiratório forçado no primeiro segundo $\left(\mathrm{FEV}_{1}\right)$ e o do fluxo expiratório médio (FEF 25-75\%), demonstrando importante limitação ao fluxo expiratório, o que 
caracteriza o aprisionamento aéreo. Além disso, observase maior queda da $\mathrm{SpO}_{2}$ e menor tempo de resistência ao exercício $^{(21)}$. $\mathrm{Ng}$ et al ${ }^{(22)}$ relatam que aproximadamente $44 \%$ das crianças com DBP podem apresentar sintomas e valores espirométricos sugestivos de asma.

Essas evidências demonstram alterações significativas e persistentes do crescimento das vias aéreas e do desenvolvimento alveolar durante a fase rápida de crescimento pulmonar pós-natal, com redução do diâmetro luminal das vias aéreas, resultando em persistente aumento na resistência das vias aéreas e de sibilos recorrentes ${ }^{(23)}$, podendo permanecer até a vida adulta ${ }^{(14)}$. Esses achados sugerem que, cerca de metade das crianças displásicas nascidas entre as décadas de 1980 e início da década de 1990 tenha sintomas respiratórios induzidos pelo exercício, porém com boa resposta à terapia broncodilatadora ${ }^{(21,24)}$.

As causas de indução ao broncoespasmo durante o exercício ainda são obscuras. Alguns estudos atribuem ao ar frio inspirado, à hipocapnia, à alcalose respiratória e à intensidade e duração do exercício. Atualmente, a atenção está mais voltada para o resfriamento e ressecamento do trato respiratório, que ocorre quando grandes volumes de ar seco são inspirados durante o exercício e, se associado ao aumento da FR, há maior perda de calor, aumentando os riscos de broncoespasmo ${ }^{(25)}$.

A capacidade aeróbica máxima expressa pelo consumo de oxigênio máximo $\left(\mathrm{VO}_{2 \max }\right)$ é sabidamente menor nas crianças, mas parece não ser um fator limitante para as atividades, talvez pela compensação oferecida pelo baixo peso corporal. Alguns autores ${ }^{(6,9,21)}$ encontraram valores de $\mathrm{VO}_{2 \max }$ significantemente menores no grupo de prematuros, com ou sem DBP, embora outros descrevam valores semelhantes entre nascidos prematuros e a termo ${ }^{(7,26)}$.

Outra alteração encontrada na população displásica é na razão de trocas respiratórias $(\mathrm{R})$, relação entre a produção de $\mathrm{CO}_{2}$ e o consumo de $\mathrm{O}_{2}$. Essa relação, que reflete as trocas metabólicas e gasosas durante o exercício, deve estar entre 1 e $0,7^{(25)}$. Nas crianças displásicas, a $\mathrm{R}$ é significantemente maior do que 1 , devido ao $\mathrm{CO}_{2}$ originário do ácido lático ou pela hiperventilação, uma vez que esse gás é mais solúvel no tecido do que o $\mathrm{O}_{2}$. Dessa maneira, pode-se inferir que as crianças com DBP apresentam maior nível de ácido lático durante o exercício, indicando que a oferta de oxigênio é insuficiente para a demanda, evidenciando menor capacidade de difusão de $\mathrm{O}_{2}$, com alteração significativa no desenvolvimento da superfície de trocas gasosas ${ }^{(26)}$.

\section{Respostas cardiovasculares durante o exercício}

O sistema cardiovascular é responsável pela capacidade de resposta ao aumento de demanda do organismo, frente ao exercício ou a situações adversas, como estresse e aumento do catabolismo, entre outros. Esse sistema responde pela liberação de nutrientes para o trabalho muscular, transporte dos metabólitos, termorregulação, além da manutenção do pH. Em todas as situações em que há aumento do $\mathrm{VO}_{2}$, o coração tem habilidade para aumentar sua contratilidade e, dessa forma, elevar o DC, proporcionalmente à demanda de $\mathrm{O}_{2}$.

O primeiro estudo a respeito das alterações cardiovasculares associadas ao baixo peso de nascimento surgiu ao final da década de 1980, sendo formulada a "hipótese da origem fetal"(27) para tais comprometimentos, sugerindo que os efeitos adversos ocorridos na vida uterina levariam a alterações permanentes na programação do desenvolvimento estrutural dos mecanismos cardiovasculares, o que compromete seu desenvolvimento e possibilita o surgimento de doenças como hipertensão arterial sistêmica, coronariopatias e infarto agudo do miocárdio. Desde a publicação deste estudo, muitas críticas surgiram ${ }^{(28-30)}$, pois fatores importantes como estado nutricional, restrição de crescimento intrauterino e antecedentes familiares, que influenciam as condições cardiovasculares, não foram avaliados para a formulação dessa hipótese.

Entretanto, a partir desse estudo uma série de outros trabalhos foram realizados, com o objetivo de analisar as alterações vasculares dos recém-nascidos prematuros e, principalmente, daqueles de muito baixo peso ao nascer.

\section{Resistência vascular sistêmica}

A resistência vascular sistêmica é um dos fatores importantes para o surgimento da hipertensão arterial em adultos. A redução do número de vasos periféricos e/ou aumento da espessura dos vasos causam aumento da pós-carga, necessitando de maior trabalho cardíaco e de elevação da pressão sistólica para manter ejeção adequada. Ao analisar a vasculatura periférica dos prematuros nas fases de adolescência e adulta, observaram-se alterações na microvasculatura periférica e na capacidade de aumento de fluxo sanguíneo frente a situações de isquemia, com comprometimento da microvasculaura periférica, principalmente nas mulheres e meninas que nasceram prematuras ${ }^{(29-31)}$.

A rarefação da microvasculatura nas mulheres pode ser explicada pela ação dos hormônios gonodotróficos durante a gestação e pela sua queda abrupta após o nascimento, levando 
ao aumento dos hormônios endógenos, principalmente estrógeno e progesterona, o que parece diminuir a angiogênese por alteração nos receptores da vasculatura, acelerando o processo aterosclerótico.

Já a disfunção microvascular poderia explicar as alterações de pressão arterial, principalmente sistólica, nos prematuros. Estudos demonstraram, por meio da avaliação da perfusão sanguínea após processo isquêmico, menor densidade capilar, o que predisporia à hipertensão arterial precoce, pois a espessura dos capilares periféricos causa diminuição do lúmen do vaso, aumentando a resistência ao fluxo sanguíneo ${ }^{(32)}$.

Além desses fatores, há relatos de que a hipertensão arterial sistólica em adolescentes e adultos nascidos prematuros possa estar associada à nutrição (leite materno ou fórmula) no período neonatal ${ }^{(33)}$, uso de corticoide ante e pós-natal e maior intolerância à glicose.

\section{Frequência cardíaca}

A frequência cardíaca (FC) é um importante fator para a manutenção do DC e sabe-se que na criança em repouso, quando comparada ao adulto, a FC é maior, decrescendo com o aumento da idade ${ }^{(34)}$. Essa maior atividade cronotrópica deve-se a um mecanismo compensatório relacionado ao menor volume do coração, menor volume sanguíneo e, consequentemente, menor volume de ejeção ${ }^{(4)}$. Além disso, há maior ativação dos quimiorreceptores periféricos, devido ao menor recrutamento de fibras musculares para uma mesma demanda de trabalho.

Em crianças nascidas a termo de diferentes faixas etárias, a FC máxima atingida durante o exercício é sempre acima dos 180 batimentos por minuto, sendo comuns valores de 200 batimentos por minuto no pico máximo ${ }^{(34,35)}$. Fisiologicamente, o aumento da intensidade do esforço e da demanda metabólica aumentam progressivamente e proporcionalmente à FC, tanto em adultos quanto em crianças. Porém, os valores máximos de FC atingidos e a correlação entre a FC máxima e o alcance do $\mathrm{VO}_{2 \max }$ são maiores nas crianças do que nos adultos ${ }^{(34,36)}$.

Nas crianças prematuras, principalmente as portadoras de DBP, a FC máxima é significantemente menor do que nas crianças normais ${ }^{(26,37-39)}$. As razões pelas quais a criança prematura apresenta menor FC ainda são obscuras, mas associa-se às alterações apresentadas com o parto prematuro como menor distensibilidade muscular do miocárdio imaturo, pobreza de organelas relacionadas ao fornecimento de cálcio (no miocárdio do feto ou do recém-nascido, a via de liberação de cálcio se dá pela troca com o sódio, diferentemente dos adultos, nos quais o cálcio já é liberado pelo retículo sarcoplasmático)e imaturidade da inervação simpática (o que não permite o acionamento de mecanismos de adaptação), levando a um desequilíbrio entre a oferta e o consumo de oxigênio ${ }^{(40)}$.

\section{Volume sistólico}

O volume sistólico representa a capacidade do coração em manter ou aumentar sua ejeção frente às elevações de pressão arterial sistêmica e maior necessidade metabólica. Em adultos, o volume sistólico se eleva em até $60 \%$ na tentativa de compensar o aumento do trabalho cardíaco e da intensidade do exercício. Nas crianças, tal resposta é atenuada ${ }^{(34)}$. Isso decorre da menor massa ventricular esquerda e menor massa corporal, com valores, mesmo ao repouso, muito inferiores aos dos adultos ${ }^{(4,34)}$. Além disso, outras possíveis razões para esse menor volume sistólico referem-se aos baixos níveis de testosterona, à menor força de contração das células cardíacas e à menor influência do sistema nervoso simpático na função cardíaca, além de níveis mais baixos de noradrenalina ${ }^{(4,34)}$. Ao comparar meninos e meninas, o volume sistólico é mais elevado em meninos devido à menor massa ventricular esquerda em meninas ${ }^{(34)}$.

\section{Pressão arterial}

De modo similar ao que ocorre no adulto, a pressão arterial sistólica (PAS) na criança aumenta de acordo com a intensidade do exercício. Os valores absolutos atingidos durante o exercício estão diretamente relacionados ao tamanho e à idade da criança, assim como ao nível pressórico em repouso ${ }^{(34,41)}$. Em relação à pressão arterial diastólica (PAD), ao contrário do observado nos adultos, há diminuição do seu valor basal durante o exercício, embora existam estudos que contradigam essa informação ${ }^{(34)}$.

A pressão média permanece inalterada durante o exercício dinâmico devido a alguns efeitos imediatos ocasionados pela estimulação dos beta-receptores, que desencadeiam diminuição da resistência periférica e a taquicardia sinusal ${ }^{(25)}$.

Crianças da raça negra apresentam respostas mais exacerbadas da pressão arterial durante o exercício do que crianças caucasianas, devido à maior resistência vascular periférica encontrada naquelas ${ }^{(25,34)}$. Em relação às crianças que nasceram prematuras, os valores de pressão arterial sistólica são, em média, $2-5 \mathrm{mmHg}$ mais elevados do que o de nascidas a termo, havendo uma relação inversa entre a idade gestacional e os valores de pressão $\operatorname{arterial}^{(29,30)}$.

Durante a adolescência e a fase adulta de indivíduos com histórico de prematuridade, principalmente os que evoluíram com DBP, a PAS é mais elevada do que na população em geral, mesmo em repouso. Esse achado pode associar-se ao 
menor crescimento uterino e às alterações neuro-humorais que regulam o aumento de catecolaninas e de angiotensina ${ }^{(42)}$. Estudos de coorte que incluíram mais de 400 adultos com histórico de prematuridade, comparados a adultos nascidos a termo, observaram que as mulheres nascidas prematuras são mais predispostas à hipertensão arterial diastólica ${ }^{(43,44)}$, maiores valores de PAS ${ }^{(26,30,45,46)}$ e apresentam maior incidência de problemas renais ${ }^{(43,44)}$.

Outros estudos têm questionado a influência da nutrição intra e extrauterina para o desenvolvimento cardiovascular, pois, em crianças nascidas pequenas para a idade gestacional, ou seja, com baixo ganho ponderal durante a gestação e restrição do crescimento intrauterino, há maior incidência de hipertensão arterial, provavelmente causada pela diferença na espessura endotelial e na tonicidade dos vasos dessas crianças ${ }^{(47-49)}$, o que contribui para o aumento da resistência vascular sistêmica. Esse comprometimento é mais evidente ao se comparar gemelares com síndrome feto-fetal, uma vez que o doador tem menor trama vascular periférica que o receptor, propiciando maior elevação da pressão $\operatorname{arterial}^{(50)}$.

A recuperação nutricional por meio da aceleração do desenvolvimento durante a infância, chamado catch up, parece também influenciar a pressão arterial, observando-se maiores valores de PAS nesses prematuros quando o fenômeno de $c$ atch $u p$ ocorre de forma acelerada ${ }^{(51)}$.

\section{Débito cardíaco}

O débito cardíaco (DC) é o fator mais importante a ser considerado em relação à circulação, tendo como determinantes para sua alteração a FC, o volume sistólico e a resistência vascular sistêmica. Fisiologicamente, o aumento crescente da intensidade do exercício causa elevação proporcional do DC. Esse mecanismo ocorre nos adultos e em crianças; entretanto, é proporcionalmente menor nas crianças ${ }^{(34,35)}$, devido à presença de menor volume cardíaco e sistólico e maior diferença arteriovenosa de $\mathrm{O}_{2}$. Apesar dessas diferenças, o DC é suficiente para compensar o transporte de oxigênio nos pacientes pediátricos ${ }^{(4,34,35)}$.

\section{Referências bibliográficas}

1. Northway WH Jr. Bronchopulmonary dysplasia: thirty-three years later. Pediatr Pulmonol 2001; (Suppl 23):5-7.

2. Jobe $A H$, Ikegami M. Prevention of bronchopulmonary dysplasia. Curr Opin Pediatr 2001;13:124-9.

3. Coalson JJ. Pathology of new bronchopulmonary dysplasia. Semin Neonatol 2003;8:73-81.
As possíveis razões para essas diferenças podem estar relacionadas ao fluxo sanguíneo mais elevado nos músculos periféricos das crianças em relação aos adultos e maior vasodilatação das artérias que incorporam os músculos, seja em crianças a termo ou prematuras, com ou sem $\mathrm{DBP}^{(9)}$. Apesar dos mecanismos compensatórios na criança, o menor DC pode ser desfavorável durante o esforço máximo, quando a extração periférica de oxigênio está esgotada ou quando a criança é exposta a estresses adicionais durante o esforço, calor, altitude ou hipóxia ${ }^{(25,34,35)}$.

Especificamente em relação ao grau de atividade física atingida, vários estudos demonstram que indivíduos nascidos prematuros têm a mesma aptidão física que os nascidos a termo ${ }^{(7,9,26,38)}$, embora outros demonstrem que crianças e adolescentes exprematuros, independentemente de terem apresentado DBP e/ou SDR, mostram menor capacidade física que os de mesma idade nascidos a termo ${ }^{(21,37)}$. Para outros autores, a baixa capacidade física do prematuro estaria associada à falta de treinamento ${ }^{(52)}$.

\section{Considerações finais}

As consequências da prematuridade em longo prazo ainda estão sendo pesquisadas. Poucos estudos foram realizados a respeito das respostas cardiovasculares ao exercício e se o treinamento poderia alterar o quadro tanto cardíaco quanto pulmonar.

A maior parte das publicações refere-se a crianças nascidas entre as décadas de 1970 e início da década de 1990, em um período no qual o uso de corticoide antenatal e a terapia com surfactante exógeno não existiam ou eram pouco utilizados. Entretanto, os estudos longitudinais mais recentes, realizados em crianças que receberam corticoide antenatal e surfactante pós-natal, mostram tendência de que essas restrições na capacidade física persistam, sugerindo que as alterações fisiológicas ocorridas pelo nascimento prematuro possam ser um fator mais relevante.

Acredita-se que a reabilitação pulmonar nessa população contribuiria para otimizar seu condicionamento e reduziria suas limitações, melhorando sua qualidade de vida.

4. Prado DM, Dias RG, Trombetta IC. Comportamento das variáveis cardiovasculares, ventilatórias e metabólicas durante o exercício: diferenças entre crianças e adultos. Arq Bras Cardiol 2006;87:e149-55.

5. Rowland TW, Cunningham LN. Development of ventilatory responses to exercise in normal white children. A longitudinal study. Chest 1997; 111:327-32. 
6. Santuz P, Baraldi E, Zaramella P, Filippone M, Zacchello F. Factors limiting exercise performance in long-term survivors of bronchopulmonary dysplasia. Am J Respir Crit Care Med 1995;152:1284-9.

7. Jacob SV, Lands LC, Coates AL, Davis GM, MacNeish CF, Hornby L et al. Exercise ability in survivors of severe bronchopulmonary dysplasia. Am J Respir Crit Care Med 1997;155:1925-9.

8. Welsh L, Kirkby J, Lum S, Odendaal D, Marlow N, Derrick G et al. The EPICure study: maximal exercise and physical activity in school children born extremely preterm. Thorax 2010;65:165-72.

9. Kriemler S, Keller H, Saigal S, Bar-Or O. Aerobic and lung performance in premature children with and without chronic lung disease of prematurity. Clin J Sport Med 2005;15:349-55.

10. Wong PM, Lees AN, Louw J, Lee FY, French N, Gain K et al. Emphysema in young adult survivors of moderate-to-severe bronchopulmonary dysplasia. Eur Respir J 2008;32:321-8.

11. Robin B, Kim YJ, Huth J, Klocksieben J, Torres M, Tepper RS et al. Pulmonary function in bronchopulmonary dysplasia. Pediatr Pulmonol 2004;37:236-42.

12. Hülskamp G, Lum S, Stocks J, Wade A, Hoo AF, Costeloe K et al. Association of prematurity, lung disease and body size with lung volume and ventilation inhomogeneity in unsedated neonates: a multicentre study. Thorax 2009;64:240-5.

13. Bhutani VK, Abbasi S. Long-term pulmonary consequences in survivors with bronchopulmonary dysplasia. Clin Perinatol 1992;19:649-71.

14. Narang I, Rosenthal M, Cremonesini D, Silverman M, Bush A. Longitudinal evaluation of airway function 21 years after preterm birth. Am J Respir Crit Care Med 2008;178:74-80

15. Eber E, Zach MS. Long term sequelae of bronchopulmonary dysplasia (chronic lung disease of infancy).Thorax 2001;56:317-23.

16. Baraldi E, Filippone M. Chronic lung disease after premature birth. N Engl J Med 2007;357:1946-55.

17. Talmaciu I, Ren CL, Kolb SM, Hickey E, Panitch HB. Pulmonary function in technology-dependent children 2 years and older with bronchopulmonary dysplasia. Pediatr Pulmonol 2002;33:181-8.

18. Hofhuis W, Huysman MW, van der Wiel EC, Holland WP, Hop WC, Brinkhorst $G$ et al. Worsening of V'maxFRC in infants with chronic lung disease in the first year of life: a more favorable outcome after high-frequency oscillation ventilation. Am J Respir Crit Care Med 2002;166:1539-43.

19. McLeod A, Ross P, Mitchell S, Tay D, Hunter L, Hall A et al. Respiratory health in a total very low birthweight cohort and their classroom controls. Arch Dis Child 1996;74:188-94.

20. Filippone M, Sartor M, Zacchello F, Baraldi E. Flow limitation in infants with bronchopulmonary dysplasia and respiratory function at school age. Lancet 2003;361:753-4.

21. Kilbride HW, Gelatt MC, Sabath RJ. Pulmonary function and exercise capacity for ELBW survivors in preadolescence: effect of neonatal chronic lung disease. J Pediatr 2003;143:488-93.

22. Ng DK, Lau WY, Lee SL. Pulmonary sequelae in long-term survivors of bronchopulmonary dysplasia. Pediatr Int 2000;42:603-7.

23. Mello RR, Dutra MV, Lopes JM. Respiratory morbidity in the first year of life of preterm infants discharged from a neonatal intensive care unit. J Pediatr (Rio J) 2004;80:503-10.

24. Sadeghi H, Lowenthal DB, Dozor AJ. Inspiratory flow limitation in children with bronchopulmonary dysplasia. Pediatr Pulmonol 1998;26:167-72.

25. Powers SK, Howley ET, editors. Fisiologia do exercício: teoria e aplicação ao condicionamento e ao desempenho. $3^{\mathrm{a}}$ ed. São Paulo: Manole; 2000.

26. Abreu LR, Costa-Rangel RC, Gastaldi AC, Guimarães RC, Cravo SL, Sologuren MJ. Avaliação da aptidão cardiorrespiratória de crianças com displasia broncopulmonar. Rev Bras Fisioter 2007;11:105-11.

27. Barker DJ. Fetal origins of coronary heart disease. BMJ 1995;311:171-4.

28. Irving RJ, Belton NR, Elton RA, Walker BR. Adult cardiovascular risk factors in premature babies. Lancet 2000;355:2135-6.

29. Siewert-Delle A, Ljungman S. The impact of birth weight and gestational age on blood pressure in adult life: a population-based study of 49-year-old men. Am J Hypertens 1998;11:946-53.

30. Kistner A, Celsi G, Vanpee M, Jacobson SH. Increased blood pressure but normal renal function in adult women born preterm. Pediatr Nephrol 2000;15:215-20.

31. Bonamy AK, Bendito A, Martin H, Andolf E, Sedin G, Norman M. Preterm birth contributes to increased vascular resistance and higher blood pressure in adolescent girls. Pediatr Res 2005;58:845-9.

32. Cheung YF, Wong KY, Lam BC, Tsoi NS. Relation of arterial stiffness with gestational age and birth weight. Arch Dis Child 2004;89:217-21.

33. Singhal A, Cole TJ, Lucas A. Early nutrition in preterm infants and later blood pressure: two cohorts after randomised trials. Lancet 2001;357:413-9.

34. Turley KR. Cardiovascular responses to exercise in children. Sports Med 1997;24:241-57.

35. Turley KR, Wilmore JH. Cardiovascular responses to treadmill and cycle ergometer exercise in children and adults. J Appl Physiol 1997;83:948-57.

36. Karila C, de Blic J, Waernessyckle S, Benoist MR, Scheinmann P. Cardiopulmonary exercise testing in children: an individualized protocol for workload increase. Chest 2001;120:81-7.

37. Smith LJ, van Asperen PP, McKay KO, Selvadurai H, Fitzgerald DA. Reduced exercise capacity in children born very preterm. Pediatrics 2008;122:e287-93.

38. Sritippayawan S, Harnruthakorn C, Deerojanawong J, Samransamruajkit R, Prapphal N. Optimal level of physical activity in children with chronic lung diseases. Acta Paediatr 2008;97:1582-7.

39. Pianosi PT, Fisk M. Cardiopulmonary exercise performance in prematurely born children. Pediatr Res 2000;47:653-8.

40. Santana MV, editor. Cardiopatias congênitas no recém-nascido - diagnóstico e tratamento. $2^{\text {a }}$ ed. São Paulo: Atheneu; 2005.

41. Wanne OP, Haapoja E. Blood pressure during exercise in healthy children. Eur J Appl Physiol Occup Physiol 1988;58:62-7.

42. Abman S. Monitoring cardiovascular function in infants with chronic lung disease of prematurity. Arch Dis Child Fetal Neonatal Ed 2002;87:F15-8.

43. Hack M, Schluchter M, Cartar L, Rahman M. Blood pressure among very low birth weight ( $<1.5 \mathrm{~kg}$ ) young adults. Pediatr Res 2005;58:677-84.

44. Irving RJ, Shore AC, Belton NR, Elton RA, Webb DJ, Walker BR. Low birth weight predicts higher blood pressure but not dermal capillary density in two populations. Hypertension 2004;43:610-3.

45. Keijzer-Veen MG, Finken MJ, Nauta J, Dekker FW, Hille ET, Frölich M et al. Is blood pressure increased 19 years after intrauterine growth restriction and preterm birth? A prospective follow-up study in The Netherlands. Pediatrics 2005;116:725-31.

46. Doyle LW, Faber B, Callanan C, Morley R. Blood pressure in late adolescence and very low birth weight. Pediatrics 2003;111:252-7.

47. Walker SP, Gaskin P, Powell CA, Bennett FI, Forrester TE, GranthamMcGregor S. The effects of birth weight and postnatal linear growth retardation on blood pressure at age 11-12 years. J Epidemiol Community Health 2001;55:394-8.

48. Singhal A, Kattenhorn M, Cole TJ, Deanfield J, Lucas A. Preterm birth, vascular function, and risk for atherosclerosis. Lancet 2001;358:1159-60.

49. Johansson S, lliadou A, Bergvall N, Tuvemo T, Norman M, Cnattingius S. Risk of high blood pressure among young men increases with the degree of immaturity at birth. Circulation 2005;112:3430-6.

50. Cheung YF, Taylor MJ, Fisk NM, Redington AN, Gardiner HM. Fetal origins of reduced arterial distensibility in the donor twin in twin-twin transfusion syndrome. Lancet 2000;355:1157-8.

51. Law CM, Shiell AW, Newsome CA, Syddall HE, Shinebourne EA, Fayers PM et al. Fetal, infant, and childhood growth and adult blood pressure: a longitudinal study from birth to 22 years of age. Circulation 2002;105:1088-92.

52. Vrijlandt EJ, Gerritsen J, Boezen HM, Grevink RG, Duiverman EJ. Lung function and exercise capacity in young adults born prematurely. Am J Respir Crit Care Med 2006;173:890-6. 